(C) Cambridge University Press 2016. This is an Open Access article, distributed under the terms of the Creative Commons Attribution licence (http://creativecommons.org/licenses/by/4.0/), which permits unrestricted re-use, distribution, and reproduction in any medium, provided the original work is properly cited.

\title{
Asymmetrical reproductive interference between two sibling species of tea looper: Ectropis grisescens and Ectropis obliqua
}

\author{
G.-H. Zhang ${ }^{1,2}$, Z.-J. Yuan ${ }^{1}$, K.-S. Yin ${ }^{1}$, J.-Y. Fu' ${ }^{1}$, M.-J. Tang ${ }^{1}$ \\ and Q. Xiao ${ }^{1 *}$ \\ ${ }^{1}$ Tea Research Institute, Chinese Academy of Agricultural Sciences, \\ Hangzhou, 310008, China: ${ }^{2}$ South China Botanical Garden, Chinese \\ Academy of Sciences, Guangzhou, 510650, China
}

\begin{abstract}
Ectropis grisescens Warren and Ectropis obliqua (Prout) are two morphologically similar sibling species with overlapping ranges. In this study, manipulative laboratory experiments were conducted to examine the possibility of reproductive interference in sympatric populations of E. grisescens and E. obliqua and the potential consequences of the mating interaction. Our results showed that the presence of males or females of different species could incur mating interference and significant reduction of $F_{1}$ offspring. The reduction was not significant relevant to the initial relative abundance of E. grisescens and E. obliqua. Detailed observations of mating opportunity showed that female mating frequencies of both species were not significantly affected by the absolute species density, but the mating success of E. obliqua females with conspecific males depended on species ratio. In addition, adding males to the other species resulted in lower number of offspring suggesting that the males' behaviour might be linked with mating interference. Males of both E. grisescens and E. obliqua could interfere the intraspecific mating of the other species, but the impact of the mating interference differed. These combined data indicated that asymmetric reproductive interference existed in E. grisescens and E. obliqua under laboratory conditions, and the offspring of the mixed species were significantly reduced. The long term outcome of this effect is yet to be determined since additional reproductive factors such as oviposition rate and progeny survival to adulthood may reduce the probability of demographic displacement of one species by the other in overlapping niches.
\end{abstract}

Keywords: tea looper, sibling species, reproductive interference, mating interaction, species exclusion

(Accepted 27 June 2016)

\section{Introduction}

Ectropis grisescens Warren and Ectropis obliqua (Prout) (Lepidoptera: Geometridate: Ennominae) feed on tea leaves

*Address for correspondence

Tel: +860571 86650801

Fax: +86 057186650720

E-mail: xqtea@mail.tricaas.com and tender buds. They infest thousands of hectares of tea a year, severely reducing growth, and impacting the tea production in the following year (Zhang \& Tan, 2004; Zhang et al., 2014). E. grisescens has been reported from Anhui, Fujian, Henan, Hunan, Hubei, Jiangxi and Zhejiang provinces in China (Jiang et al., 2014). It is probably indigenous to China, as there are no reports of E. grisescens abroad (Warren, 1894; Swinhoe, 1902; Wehrli, 1939-1954). E. obliqua was first found and named in Japan, and now in Korea and in China (Wehrli, 1939-1954; Sato, 1984; Kim et al., 2001). E. obliqua 
has been reported from Anhui, Jiangsu and Zhejiang provinces and Shanghai city in China (Jiang et al., 2014). E. grisescens and E. obliqua are morphologically similar and hard to discriminate in tea plantations. Because of these similarities, E. obliqua is likely mistaken for a variant of E. grisescens as it was when reported for the first time (Warren, 1894).

Despite their morphological similarities, E. obliqua and E. grisescens are two sibling species in the same genus in China (Jiang et al., 2014; Zhang et al., 2014). Our previous research of tea loopers from 17 tea fields in different parts of China shows that the two species, although being morphologically similar, had clear reproductive isolation and variable mitochondrial cytochrome oxidase I (COI) sequences (Zhang et al., 2014). Recently, by microscopic examination of some morphological traits can separate the two species. In additional, a genetic distance between E. obliqua and E. grisescens, based on COI sequences, of about 3.7\% (Jiang et al., 2014). These phylogenetic analyses revealed that E. obliqua and E. grisescens COI sequences fall into two taxonomic clades, and form separate monophyletic groups (Jiang et al., 2014; Zhang et al., 2014). Previous attempts to cross E. grisescens and E. obliqua did produce fewer female offspring than intraspecies mating, which resulted in an unbalanced sex ratio in the hybrid $F_{1}$ generation. The hatching rate, survival to adult, and percent of normal adults was much lower than with intra-species mating. Moreover, a self-cross of $F_{1}$ generation adults produced infertile eggs or no eggs (Xi et al., 2014; Zhang et al., 2014). These findings can be explained as closely related species often have incompletely isolated mate recognition systems (Reitz \& Trumble, 2002). Laboratory experiments have shown that E. grisescens and E. obliqua are capable of producing viable $F_{1}$ hybrids but that these females are sterile as their $F_{2}$ generation was not viable (hybrid breakdown), indicating, most likely, a post-zygotic reproductive barrier (Xi et al., 2014; Zhang et al., 2014).

Asymmetric mating behaviour of both males and females may lead to different consequences in reproductive performance contributing to facilitation or prevention of competitive displacement of indigenous species by invaders (Scott Schneider et al., 2004; Crowder et al., 2010a, b; Hochkirch \& Lemke, 2011; Chapple et al., 2012; Luan \& Liu, 2012; Wang et al., 2012; Luan et al., 2013). Such sexual interaction plays a critical role in biological invasion and has been called reproductive interference. Reproductive interference is defined as any kind of interspecific sexual interaction, caused by incomplete species recognition systems, which can reduce the fitness of individuals of at least one of the species involved (Gröning \& Hochkirch, 2008). Reproductive interference is one of the major factors mediating species exclusion among insects (Reitz \& Trumble, 2002). Reproductive interference is generally asymmetric (one species is affected more than the other) and is positively frequency-dependent (Gröning \& Hochkirch, 2008; Kishi et al., 2009). Reproductive interference can affect the population dynamics, abundance, habitat choice, geographical distribution of the species involved, is more likely to lead to species exclusion or niche partitioning (Gröning et al., 2007; Hochkirch et al., 2007; Liu et al., 2007; Gröning \& Hochkirch, 2008; Kishi et al., 2009; Luan et al., 2014; Sun et al., 2014). Studies on such interspecific interactions in species can give information about the maintenance of speciation, as they might drive speciation due to reinforcement of pre-mating barriers (Servedio \& Noor, 2003; Seehausen, 2004; Keranen et al., 2013). However, the ecological significance of reproductive interference has mostly been discussed in the context of biological invasions, and is still often undervalued.

Previously we found that E. grisescens and E. obliqua occur in separate tea plantations, despite being reported from the same provinces (Zhang et al., 2014). Here additional collections were undertaken and mixed populations of two species were found in Hangzhou, Wuhan, Xinyang, and Nanchang (Jiang et al., 2014; partial data is not published). Previous studies indicated that interspecific sexual interaction (reproductive interference) might explain this distribution pattern (e.g. Gröning et al., 2007; Hochkirch et al., 2007; Thum, 2007; Noriyuki et al., 2012). In this study, we tested whether asymmetric reproductive interference occurs in E. grisescens and E. obliqua when they co-exist under laboratory conditions and examined the reproductive consequences of these interferences, and whether reproductive success is dependent in the abundances of sexual interacting species.

\section{Materials and methods}

\section{Collection and culturation of E. grisescens and E. obliqua}

The population of E. grisescens used in this study was collected in 2012 from Songyang $\left(119^{\circ} 42^{\prime} \mathrm{E}, 2^{\circ} 49^{\prime} \mathrm{N}\right)$, Zhejiang; whereas the population of E. obliqua was obtained from Yuhang $\left(119^{\circ} 90^{\prime} \mathrm{E}, 30^{\circ} 39^{\prime} \mathrm{N}\right)$, Zhejiang, China. All species were maintained under the same conditions in the laboratory of the Plantation Centre of Tea Research Institute of Chinese Academy of Agricultural Sciences (CAAS), Hangzhou, China. Cultures of both species were fed with tea fresh leaves in laboratory conditions for a year. All the experiments were conducted at $24 \pm 1^{\circ} \mathrm{C}, 13: 11 \mathrm{~h}(\mathrm{~L}: \mathrm{D})$ and $75 \pm 5 \%$ relative humidity (RH).

\section{Collection of newly emerged virgin adults}

In order to ensure that the adults used were virgin, a few days prior to experiments, tea looper pupae from each culture were collected singly and their sex determined using a stereo microscope before adults emerged. The male and female pupae of each population were placed singly into transparent, colourless glass cages. Newly emerged adults were randomly chosen and prepared for experiments.

\section{Crossing experiments between $\mathrm{E}$. grisescens and E. obliqua}

Crossing experiments were conducted in transparent, colourless glass cages with three combinations of the two species (table 1): (i) E. obliqua in single culture (two pairs), eight replicates; (ii) E. grisescens in single culture (two pairs), eight replicates; and (iii) E. grisescens and E. obliqua in mixed culture (one pair of each species), twelve replicates. All the females were allowed to lay eggs for 5 days and then removed. Females that did not lay eggs during this period, or females that laid eggs that did not hatch in 10 days, were considered sterile. The eggs laid by each female in a replicate were calculated by the total eggs weight divided by average egg weight. Then the number of developing larvae and pupae, and emerged adults was recorded every $24 \mathrm{~h}$ for 30-35 days. These records were used to calculate the rates of hatching, pupation and emergence, the proportions of normal adults, and the female progeny ratios. 
Table 1. Number of $F_{1}$ eggs laid, their hatching, pupation, emergence rate and female progeny ratio, in crossing experiments of $E$. grisescens and E. obliqua.

\begin{tabular}{lrccccc}
\hline Treatments & $N^{*}$ & Number of eggs laid/female & Hatching rate & Pupation rate & Emergence rate & Female progeny ratio \\
\hline E. grisescens + E. grisescens & 8 & $424 \pm 8.35 \mathrm{a}$ & $0.67 \pm 0.02 \mathrm{a}$ & $0.94 \pm 0.01 \mathrm{a}$ & $0.81 \pm 0.03 \mathrm{a}$ & $0.46 \pm 0.04 \mathrm{a}$ \\
E. obliqua + E. obliqua & 8 & $391 \pm 11.32 \mathrm{a}$ & $0.72 \pm 0.02 \mathrm{a}$ & $0.91 \pm 0.02 \mathrm{a}$ & $0.88 \pm 0.03 \mathrm{a}$ & $0.53 \pm 0.03 \mathrm{a}$ \\
E. grisescens + E. obliqua & 12 & $392 \pm 15.42 \mathrm{a}$ & $0.48 \pm 0.07 \mathrm{~b}$ & $0.91 \pm 0.02 \mathrm{a}$ & $0.70 \pm 0.08 \mathrm{a}$ & $0.40 \pm 0.06 \mathrm{a}$ \\
\hline
\end{tabular}

$N^{*}$, number of replicates.

Data in the table are mean $\pm S E$, and means on the same line followed by different letters indicate significant differences $(P<0.05)$.

Table 2. Number of $F_{1}$ eggs laid, their hatchability, progeny survival to adulthood and female progeny ratio, in reproductive interference with different mix of E. grisescens and E. obliqua.

\begin{tabular}{|c|c|c|c|c|c|}
\hline Treatments & $N^{*}$ & $\begin{array}{l}\text { Number of eggs } \\
\text { laid/female }\end{array}$ & $\begin{array}{l}\text { Hatching } \\
\text { rate }\end{array}$ & $\begin{array}{l}\text { Survival to } \\
\text { adult }\end{array}$ & $\begin{array}{l}\text { Female progeny } \\
\text { ratio }\end{array}$ \\
\hline $\begin{array}{l}(1 \text { E. obliqua }+1 \text { E. obliquað })+(1 \text { E. grisescenso }+1 \\
\text { E. grisescenso })\end{array}$ & 8 & $379.14 \pm 18.64 a$ & $0.46 \pm 0.089 a$ & $0.66 \pm 0.071 \mathrm{a}$ & $0.41 \pm 0.073 a$ \\
\hline $\begin{array}{l}(2 \text { E. obliqua }+2 \text { E. obliquað })+(1 \text { E. grisescenso }+1 \\
\left.\text { E. grisescens } \sigma^{\star}\right)\end{array}$ & 8 & $313.50 \pm 19.91 b$ & $0.64 \pm 0.049 a$ & $0.69 \pm 0.055 a$ & $0.46 \pm 0.039 a$ \\
\hline
\end{tabular}

$N^{*}$, number of replicates.

Data in the table are mean $\pm \mathrm{SE}$, and means on the same line followed by different letters indicate significant differences $(P<0.05)$.

\section{Mating interactions in mixed cultures with E. grisescens and $\mathrm{E}$. obliqua}

In order to study the difference in offspring in the mixed of E. grisescens and E. obliqua, three treatments were conducted with different initial relative abundance for each of the two species in transparent colourless glass cages (table 2). One pair of one species adults was supplemented with one or two pairs of the same or the other species. Eight replicates were used for each treatment. Number of eggs, larvae, pupae and adults were counted. The hatching rate, progeny survival to adulthood and female progeny in $F_{1}$ were calculated.

\section{Mating opportunity of E. grisescens and E. obliqua}

In order to study whether the presence of individuals of different species affected female mating opportunity with males of the same species, three independent treatments with different proportions of species were compared in transparent colourless glass cages: (iv) only a single conspecific couple were introduced into a cage (number of the conspecific pairs : number of heterospecific pairs $=1: 0)$; $(\mathrm{v})$ one pair of each species in each replicate were introduced into a cage (1:1) and (vi) two pairs of one species and one pair of the other species were introduced into a cage (2:1 or $1: 2)$. The proportion of mated females was compared among the three treatments. The species were discriminated by the marked wings of male adults between individuals. Each treatment started in the middle of the day and we recorded whether intraspecific (conspecific) and interspecific (heterospecific) mating occurred in the evening. Eight replications were arranged for each trial.

\section{Mating interference in males of E. grisescens and E. obliqua}

In order to test whether male behaviour is responsible for mating interference, two parallel treatments were conducted.
Changes in the mean number of intraspecific mating and production of progeny for the first 5 days after emergence were recorded after a pair of E. obliqua $\delta \times q$ was respectively supplemented with one, two and three virgin males of E. obliqua or E. grisescens. The same experiment was conducted with a pair of $E$. grisescens $\delta \times$ o supplemented with one to three virgin males of E. grisescens or E. obliqua. The experiment was replicated 8 times for each combination. We recorded if successful mating occurred in the first evening. Number of eggs, larvae, pupae and adults, and female progeny in $F_{1}$ were determined as described in the crossing experiments.

\section{Data analysis}

For crossing experiments between E. grisescens and E. obli$q u a$, some parameters were compared among treatments using one-way analysis of variance (ANOVA) and means were separated by Duncan's multiple range test.

For mating interactions in mixed cultures with E. grisescens and E. obliqua, data were analysed by one-way ANOVA and Duncan's multiple range tests of variance.

For mating opportunity of E. grisescens and E. obliqua, mating success of individual female from each species was analyzed by using a generalized linear model (GLM) with a binomial error structure that indicated whether each individual female was successfully mated (no mating $=0$, mating $=1$ ). Absolute species density (number of conspecific pairs/cage) and relative species density (number of conspecific pairs/ total number of species in cage) were represented as main effects.

For mating interference in males of E. grisescens and E. obliqua, all parameters were analyzed using by one-way ANOVA followed by Duncan's multiple range test, and percentages were transformed by arcsine square root before analysis.

All statistical analyses were done using the IBM SPSS statistical software version 21.0. 

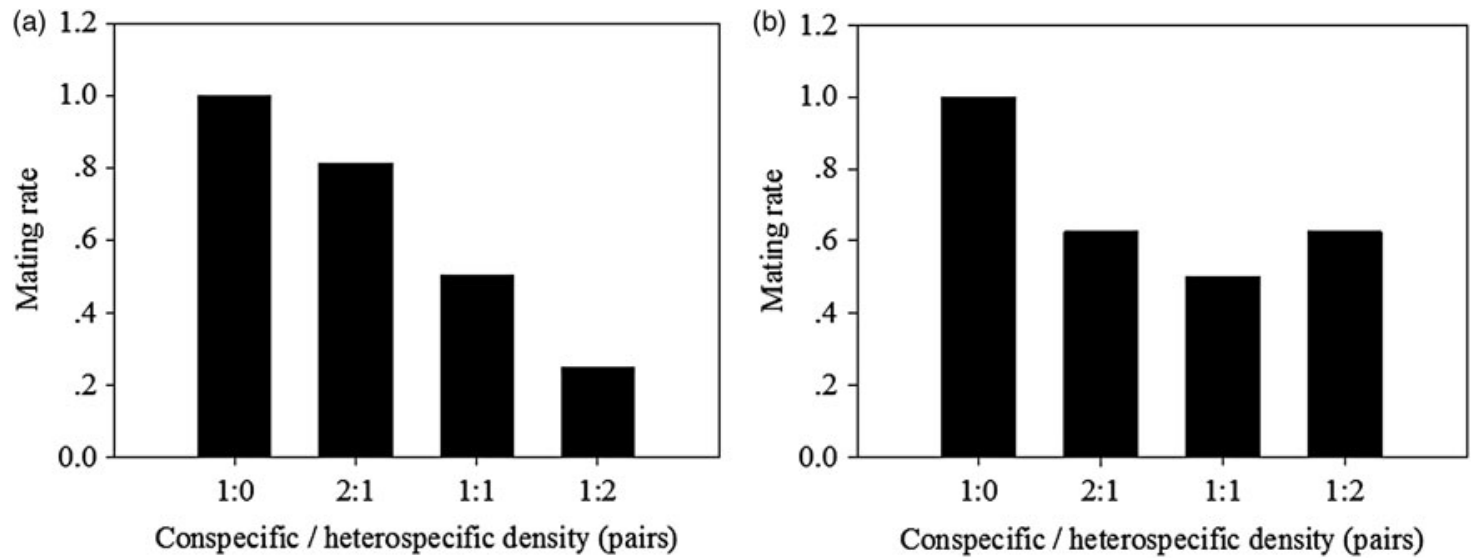

Fig. 1. Mating rate in relation to relative species density in (a) E. obliqua and (b) E. grisescens females.

\section{Results}

Crossing experiments between $\mathrm{E}$. grisescens and E. obliqua

The presence of males and females of different species was found to disturb the reproduction of mated females (table 1). While there was no significant difference in the number of eggs laid per female of each treatment $\left(F_{2,24}=1.921, P=0.168\right)$, significant hatching reduction was observed in mixed cultures $\left(F_{2,24}=6.398, P=0.006\right)$. The pupation rate and emergence rate of $F_{1}$ were both lower in mixed cultures than that in pure culture, even these parameters were not significantly different between pure and mixed cultures $\left(F_{2,25}=0.640\right.$, $\left.P=0.536 ; F_{2,25}=2.109, P=0.142\right)$. Moreover, there was no significant difference in female ratio in $F_{1}\left(F_{2,25}=0.579, P=0.568\right)$, the female ratio of $F_{1}$ was less than that from pure parents. Therefore, the less $F_{2}$ progeny were produced.

\section{Mating interactions in mixed cultures with $\mathrm{E}$. grisescens and $\mathrm{E}$. obliqua}

Comparisons among all the three treatments indicated there were no significant differences in the hatching rate of $F_{1}\left(F_{2,21}=1.498, P=0.246\right)$, survival to adult $\left(F_{2,21}=0.110\right.$, $P=0.896)$ and female progeny ratio of the $F_{1}\left(F_{2,21}=0.481\right.$, $P=0.625)$, although these parameters were lower when $E$. grisescens and E. obliqua equally presented (table 2). Significantly fewer eggs were observed in the third treatment (two pairs of E. obliqua and one pair of E. grisescens) compared with the other two treatments $\left(F_{2,21}=4.426, P=0.025\right)$.

\section{Mating opportunity of E. grisescens and E. obliqua}

Next, we tested the mating opportunities of two species. The mating rate of E. obliqua females was not affected by the absolute species density, but was significantly affected by the relative species density in the given treatment (fig. 1a, GLM, absolute density, the parameter estimate $\pm \mathrm{SE}=$ $3.17 \pm 3.44$, d.f. $=1, P=0.36$; relative density, $1.93 \pm 0.97$, d.f. $=$ $1, P<0.05)$. Interestingly, mating success of intraspecific male was relatively higher in the 1:0 trial but was the lowest in the 1:2 trial. Nevertheless, E. grisescens females mating success was not affected by the absolute or relative species density (fig. 1b, GLM, absolute density, the parameter estimate $\pm \mathrm{SE}=-1.36 \pm 5.33$, d.f. $=1, P=0.70$; relative density, $0.98 \pm 0.87$, d.f. $=1, P=0.26$ )

Mating interference in males of E. grisescens and E. obliqua

We found an influence of additional E. grisescens males on reproductive success of a pair of E. obliqua, but no effect of additional E. obliqua males on E. grisescens matings. As shown in fig. 2 , the intraspecific mating rate of E. obliqua decreased with the addition of $E$. grisescens males (fig. $2 \mathrm{a} ; F_{6,49}=16.62, P<0.001$ ). Adding E. obliqua males into E. obliqua pair decreased the number of larvae slightly, however, the negative effects only became statistically significant when 3 E. grisescens males were introduced to E. obliqua pair (fig. $2 \mathrm{~b}$ ). For the total number of $F_{1}$ progeny reaching adulthood, we observed a slight, but statistically insignificant decrease in the number of $F_{1}$ adults produced by $E$. obliqua pair when 1,2 , or 3 additional E. obliqua males were present. However, adding E. grisescens males in with the E. obliqua pair reduced the number of $F_{1}$ adults produced significantly (fig. $2 c ; F_{6,49}=39.07, P<0.001$ ). The proportion of $F_{1}$ female progeny (sex ratio) was not significantly affected by any additional (1-3) E. obliqua males, while the sex ratio was significantly reduced with an increasing trend of negative influence as more E. grisescens males were added into the E. obliqua pair (fig. 2d; $\left.F_{6,49}=9.89, P<0.001\right)$.

Mating interference was also examined by introducing different numbers (1-3) of E. grisescens males or E. obliqua males into $E$. grisescens pair. We recorded the number of intraspecific mating between the two species. Results (fig. 3) showed that $E$. grisescens did not engage in interspecific mating in most cases, except for a combination of $E$. grisescens pair with one E. obliqua male (fig. 3a). This only interspecific mating group subsequently produced the lowest numbers of larvae and adults and smallest female ratio in $F_{1}$ generation, but the influence was not statistically significant. Except for this group, adding more E. obliqua males or E. grisescens males to the E. grisescens pair did not show any negative impact on mating and reproduction (fig. 3).

\section{Discussion}

Our results from the laboratory experiments revealed that reproductive interference occurs in E. grisescens and E. obliqua, and the interaction is asymmetric possibly due to different 

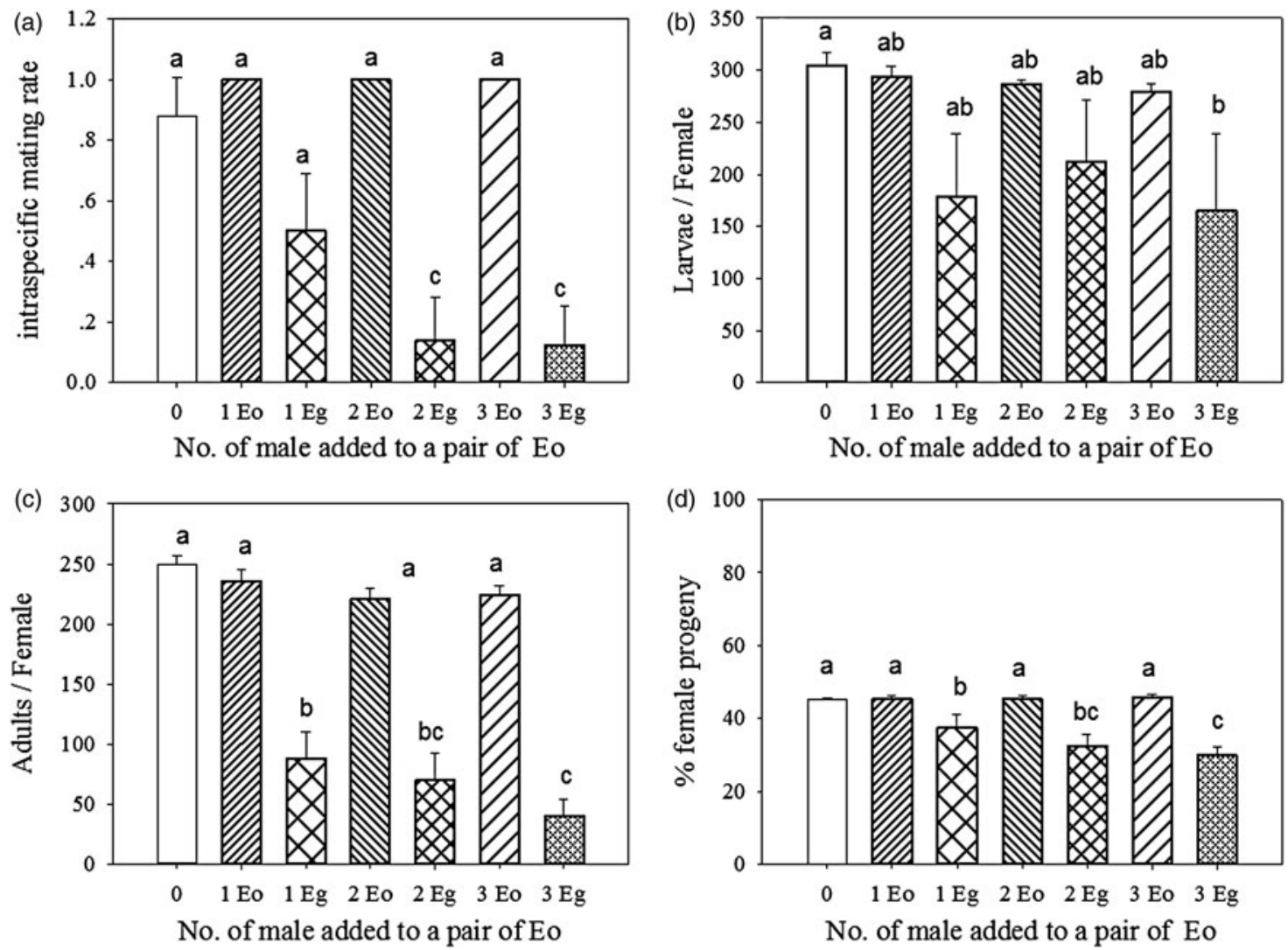

Fig. 2. E. obliqua (Eo) performance as affected by E. grisescens (Eg) males. (a) Intraspecific mating rate, (b) mean larvae, (c) mean adults and (d) proportion of female progeny within E. obliqua when a pair of E. obliqua ${ }^{\top} \times+$ was supplement with 1, 2 or 3 o of E. obliqua or E. grisescens. Error bars represent standard error of the means and different letters above bars indicate significant differences $(P<0.05)$.

mating behaviour between the two sibling species leading to different consequences of reproductive performance. In the current research, mixed populations of E. grisescens and E. obliqua were found in the tea plantations of Chun'an, Hangzhou, Wuhan, Xinyang and Nanchang. Therefore, reproductive interactions between E. grisescens and E. obliqua are likely to occur naturally because of their overlapping niches.

Studies on insects mating strategy may provide insight into the evolution of mating behaviour, competitive interactions and speciation (Rowe \& Arnqvist, 2002; Chapman et al., 2003). Females can recognize conspecific males or reject misdirected courtships from unsuitable males, and females always exert more influence over mating than males (Gröning \& Hochkirch, 2008). We observed that the frequency of E. obliqua females successfully mating with conspecific males was decreased as the proportion of individuals of the other species increased (fig. 1a). In contrast, the frequency of E. grisescens female mating was not affected by the species density ratio (fig. $1 b)$. Results from our other experiments support the conclusion of an asymmetric mating interaction between $E$. grisescens and E. obliqua. Adding more males of E. grisescens into the E. obliqua pair tend to induce more interspecific crosses $(E$. obliqua우 $\times$ E. grisescens $\left.\sigma^{\star}\right)$ (fig. 2a), but the similar phenomenon (E. grisescens $+\times E$. obliqua $\left.{ }^{\star}\right)$ was not observed in mating interaction cross between $E$. grisescens female and E. obliqua male (fig. 3a). These results suggest that males of E. grisescens have a greater ability to cause mating interference than $E$. obliqua males. As suggested above, the reduced mating success of E. obliqua in the mixed treatments may be the consequence of asymmetric mate recognition ability, e.g. females of E. grisescens were often highly sensitive in recognition and rejection males of E. obliqua, while E. obliqua females were less selective against males of $E$. grisescens. Such asymmetric mating preference phenomenon might be the result of reproductive isolation and could be a clue for the direction of evolution. Further studies will be expected to reveal the factors determining the differences in mating behaviour and mating systems that produce an asymmetric mating interaction between species.

Mate quality and number of mates are the key factor affecting female reproductive success (Bleu et al., 2012; Himuro \& Fujisaki, 2012). Our mating interference experiments suggested that mating interactions between E. grisescens and E. obliqua were frequency-dependent. When a pair of E. obliqua $\sigma \times \$$ was supplemented with different numbers of virgin males of E. grisescens, the overall number and female ratio of $F_{1}$ offspring was reduced dramatically, and the more males of E. grisescens, the fewer offspring (fig. 2). However, the overall number and female ratio of $F_{1}$ offspring did not change much when a pair of E. grisescens $0 \times q$ was supplemented with virgin males of E. obliqua (fig. 3). Mating attempts by E. grisescens males reduced the fecundity of E. obliqua females much more than mating attempts by E. obliqua males reduced the fecundity of $E$. grisescens females. Consequently, we suggest that the fecundity of females of both species would 

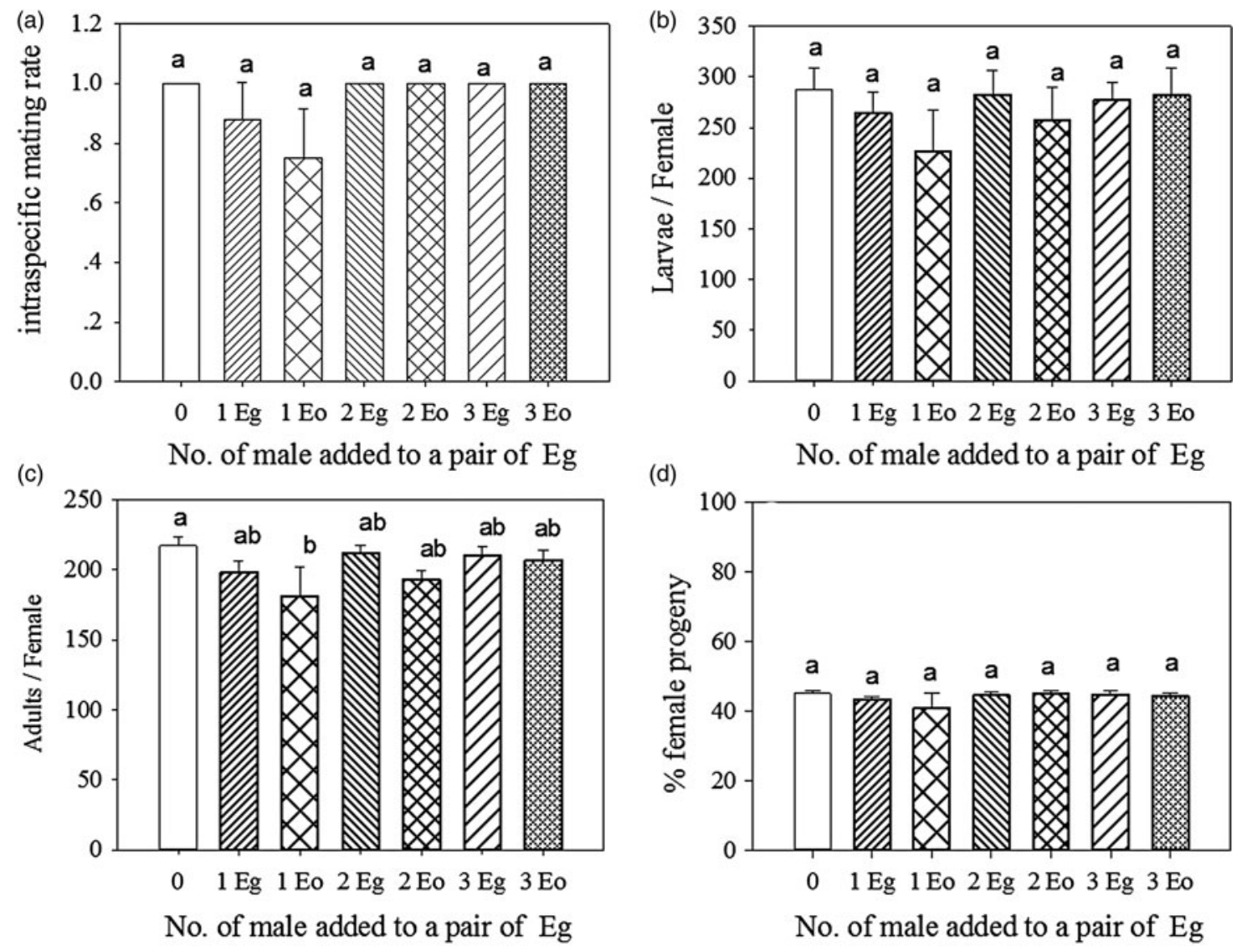

Fig. 3. E. grisescens (Eg) performance as affected by E. obliqua (Eo) males. (a) Intraspecific mating rate, (b) mean larvae, (c) mean adults and

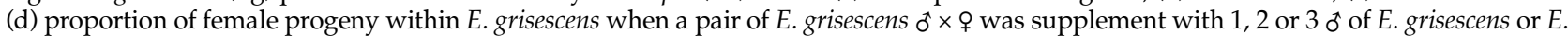
obliqua. Error bars represent standard error of the means and different letters above bars indicate significant differences $(P<0.05)$.

decrease as the number of the other species males increase, possibly due to this male frequency-dependent effect on mating success. The crossing experiments we conducted demonstrated that the presence of males and females of other species could reduce offspring sex ratio and the number of $F_{1}$ offspring (table 1). Furthermore, offspring from differently initial relative abundance of E. grisescens and E. obliqua did suffer the impact of interspecific crossing, and was less affected by the initial relative abundance (table 2 ). Overall, the presence of the other species males can affect the intraspecific mating success, and offspring were decreased after adding either male only or both males and females in laboratory.

Asymmetric mating interaction has mainly been discussed in the context of biological invasive species or hybrid zones, for example, whiteflies (Liu et al., 2007), spider mites (Ben-David et al., 2009), ground-hopper (Gröning et al., 2007), beetle (Kishi et al., 2009), and ladybird beetle (Soares \& Serpa, 2007). Although the hypothesis of species exclusion still needs to be tested experimentally, it has been suggested that reproductive interference might also offer opportunities for pest control (Ardeh et al., 2004). For example, competitive exclusion has been successfully applied in biological control of exotic Homoptera (Reitz \& Trumble, 2002). With increasing public awareness of the ecological problems of conventional insecticides, integrated pest management techniques (multiple tactics used in eradication programs) are needed (Suckling et al., 2013). Mating disruption, reducing the number of offspring, is an effective, very selective, and environmentallybenign tactic, especially for moth pests (Judd \& Gardiner, 2005; Harari et al., 2007; Suckling et al., 2013). For this reason, based on the experimental data, E. obliqua seemed to be the inferior species. E. grisescens has a stronger capacity for excluding E. obliqua than vice versa, and this capacity is associated with asymmetric reproductive interference favouring E. grisescens. The basic study of mating interference is significant for evolution of species, control of heredity, and prevention of insect pests. This study established a foundation for exploring the possibility to reduce the fitness of E. obliqua by releasing E. grisescens into E. obliqua -dominated areas.

Mating behavioural interactions between closely related species resulting from incompletely isolated mate recognition systems may lead to reproductive interference that is often asymmetric and can have serious population consequences (geographic separation) for the species (Liu et al., 2007; Gröning \& Hochkirch, 2008; Crowder et al., 2010a, b; Noriyuki et al., 2012). Reproductive interference has been commonly found under laboratory conditions, but is not always found in field conditions (Coyne \& Orr, 1989; Thum, 2007). Under natural conditions, environmental factors and several ecological mechanisms of the location might influence 
interactions between species and produce different results (Dame \& Petren, 2006; Hochkirch \& Groning, 2012). Our study shows reproductive interference in laboratory conditions and these two species with the same life history and behavioural traits have the potential for interaction in the field, but further studies are needed to determine if mating interference will occur in field conditions. Ultimately, linking detailed laboratory studies with long-term field survey datasets may provide the key to predicting patterns of species exclusion or coexistence.

\section{Acknowledgements}

The authors thank all the anonymous reviewers for their constructive comments that help improve the manuscript. We thank Eric Scott from Tufts University of USA for editorial assistance. Financial support for this study was provided by the Chinese Academy of Agricultural Sciences through Agricultural Sciences Innovation Project (CAAS-ASTIP-2014-TRICAAS), the Fundamental Research Funds of National Science and Technology (2013FY113200), and the National Science and Technology Support Program Projects (2011BAD01B02).

\section{References}

Ardeh, M.J., de Jong, P.W., Loomans, A.J.M. \& van Lenteren, C. (2004) Inter- and intraspecific effects of volatile and nonvolatile sex pheromones on males, mating behaviour, and hybridization in Eretmocerus mundus and E-eremicus (Hymenoptera : Aphelinidae). Journal of Insect Behaviour 17, 745-759.

Ben-David, T., Gerson, U. \& Morin, S. (2009) Asymmetric reproductive interference between two closely related spider mites: Tetranychus urticae and T-turkestani (Acari: Tetranychidae). Experimental and Applied Acarology 48, 213-227.

Bleu, J., Bessa-Gomes, C. \& Laloi, D. (2012) Evolution of female choosiness and mating frequency: effects of mating cost, density and sex ratio. Animal Behaviour 83, 131-136.

Chapman, T., Arnqvist, G., Bangham, J. \& Rowe, L. (2003) Sexual conflict. Trends in Ecology and Evolution 18, 41-47.

Chapple, D.G., Simmonds, S.M. \& Wong, B.B. (2012) Can behavioural and personality traits influence the success of unintentional species introductions? Trends in Ecology and Evolution 27, 57-64.

Coyne, J.A. \& Orr, H.A. (1989) Patterns of speciation in Drosophila. Evolution 43, 362-381.

Crowder, D.W., Horowitz, A.R., De Barro, P.J., Liu, S.S., Showalter, A.M., Kontsedalov, S., Khasdan, V., Shargal, A., Liu, J. \& Carriere, Y. (2010a) Mating behaviour, life history and adaptation to insecticides determine species exclusion between whiteflies. Journal of Animal Ecology 79, 563-570.

Crowder, D.W., Sitvarin, M.I. \& Carriere, Y. (2010b) Plasticity in mating behaviour drives asymmetric reproductive interference in whiteflies. Animal Behaviour 79, 579-587.

Dame, E.A. \& Petren, K. (2006) Behavioural mechanisms of invasion and displacement in Pacific island geckos (Hemidactylus). Animal Behaviour 71, 1165-1173.

Gröning, J. \& Hochkirch, A. (2008) Reproductive interference between animal species. Quarterly Review of Biology 83, 257-282.

Gröning, J., Lücke, N., Finger, A. \& Hochkirch, A. (2007) Reproductive interference in two ground-hopper species: testing hypotheses of coexistence in the field. Oikos 116, 1449-1460.
Harari, A.R., Zahavi, T., Gordon, D., Anshelevich, L., Harel, M., Ovadia, S. \& Dunkelblum, E. (2007) Pest management programmes in vineyards using male mating disruption. Pest Management Science 63, 769-775.

Himuro, C. \& Fujisaki, K. (2012) The effects of male harassment on mating duration in the seed bug, Togo hemipterus. Entomologia Experimentalis Et Applicata 142, 53-59.

Hochkirch, A. \& Groning, J. (2012) Niche overlap in allotopic and syntopic populations of sexually interacting ground-hopper species. Insect Science 19, 391-402.

Hochkirch, A. \& Lemke, I. (2011) Asymmetric mate choice, hybridization, and hybrid fitness in two sympatric grasshopper species. Behavioural Ecology and Sociobiology 65, 1637-1645.

Hochkirch, A., Gröning, J. \& Bucker, A. (2007) Sympatry with the devil: reproductive interference could hamper species coexistence. Journal of Animal Ecology 76, 633-642.

Jiang, N., Liu, S.X., Xue, D.Y., Tang, M.J., Xiao, Q., Han, H.X. (2014) External morphology and molecular identification of two tea Geometrid moth from southern China. Chinese Journal of Applied Entomology 51, 987-1002.

Judd, G.J.R. \& Gardiner, M.G.T. (2005) Towards eradication of codling moth in British Columbia by complimentary actions of mating disruption, tree banding and sterile insect technique: five-year study in organic orchards. Crop Protection 24, 718-733.

Keranen, I., Kahilainen, A., Knott, K.E., Kotiaho, J.S. \& Kuitunen, K. (2013) High maternal species density mediates unidirectional heterospecific matings in Calopteryx damselflies. Biological Journal of the Linnean Society 108, 534-545.

Kim, S.S., Beljaev, E.A., Oh, S.H. (2001) Illustrated catalogue of Geomotridae in Korea (Lepidoptera: Geometrinae, Ennominae). pp. 1-278 in Park, K.T. (Ed.). Insects of Korea Series 8. Daejeon, Korea Reasearch Institute of Bioscience and Biotechnology \& Center for Insect Systematics.

Kishi, S., Nishida, T. \& Tsubaki, Y. (2009) Reproductive interference determines persistence and exclusion in species interactions. Journal of Animal Ecology 78, 1043-1049.

Liu, S.S., De Barro, P.J., Xu, J., Luan, J.B., Zang, L.S., Ruan, Y.M. \& Wan, F.H. (2007) Asymmetric mating interactions drive widespread invasion and displacement in a whitefly. Science 318, 1769-1772.

Luan, J.B. \& Liu, S.S. (2012) Differences in mating behaviour lead to asymmetric mating interactions and consequential changes in sex ratio between an invasive and an indigenous whitefly. Integrative Zoology 7, 1-15.

Luan, J.B., De Barro, P.J., Ruan, Y.M. \& Liu, S.S. (2013) Distinct behavioural strategies underlying asymmetric mating interactions between invasive and indigenous whiteflies. Entomologia Experimentalis et Applicata 146, 186-194.

Luan, J.B., Wang, X.W., Colvin, J. \& Liu, S.S. (2014) Plant-mediated whitefly-begomovirus interactions: research progress and future prospects. Bulletin of Entomological Research 104, 267-276.

Noriyuki, S., Osawa, N. \& Nishida, T. (2012) Asymmetric reproductive interference between specialist and generalist predatory ladybirds. Journal of Animal Ecology 81, 1077-1085.

Reitz, S.R. \& Trumble, J.T. (2002) Competitive displacement among insects and arachnids. Annual Review of Entomology 47, 435-465.

Rowe, L. \& Arnqvist, G. (2002) Sexually antagonistic coevolution in a mating system: combining experimental and comparative approaches to address evolutionary processes. Evolution 56, 754-767. 
Sato, R. (1984) Taxonomic study of the genus Hypomecis Hübner and its allied genera from Japan (Lepidoptera: Geometridae: Ennominae). Special Bulletin of Essa Entomological Society 1, 1-213.

Scott Schneider, S., DeGrandi-Hoffman, G. \& Smith, D.R. (2004) The African honey bee: factors contributing to a successful biological invasion. Annual Review of Entomology 49, 351-376.

Seehausen, O. (2004) Hybridization and adaptive radiation. Trends in Ecology and Evolution 19, 198-207.

Servedio, M.R. \& Noor, M.A.F. (2003) The role of reinforcement in speciation: theory and data. Annual Review of Ecology, Evolution and Systematics 34, 339-364.

Soares, A.O. \& Serpa, A. (2007) Interference competition between ladybird beetle adults (Coleoptera : Coccinellidae): effects on growth and reproductive capacity. Population Ecology 49, 37-43.

Suckling, D.M., Stringer, L.D., Stephens, A.E., Woods, B., Williams, D.G., Baker, G. \& El-Sayed, A.M. (2013) From integrated pest management to integrated pest eradication: technologies and future needs. Pest Management Science 70, 179-189.

Sun, D.B., Li, J., Liu, Y.Q., Crowder, D.W. \& Liu, S.S. (2014) Effects of reproductive interference on the competitive displacement between two invasive whiteflies. Bulletin of Entomological Research 104, 334-346.

Swinhoe, C. (1902) New and little known species of Drepanulidae, Epiplemidae, Microniidae and Geometridae in the national collection. Transactions of the Royal Entomological Society London 3 584-677.

Thum, R.A. (2007) Reproductive interference, priority effects and the maintenance of parapatry in Skistodiaptomus copepods. Oikos 116, 759-768.

Wang, P., Crowder, D.W. \& Liu, S.S. (2012) Roles of mating behavioural interactions and life history traits in the competition between alien and indigenous whiteflies. Bulletin of Entomological Research 102, 395-405.

Warren, W. (1894) New genera and species of Geometridae. Novitates Zoologicae 1, 366-466.

Wehrli, E. (1939-1954) Subfamilie: Geometrinae. Seitz, A. (ed). Die Gross-Schmetterlinge der Erde. Abt. I. Die Spannerartigen Nachtfalter. Bd. 4 (Supplement), 254-766, pls. 19-53. Verlag, A. Kernen, Stuttgart, German.

Xi, Y., Yin, K.S., Tang, M.J., Xiao, Q. (2014) Geographic populations of the tea geometrid, Ectropis obliqua (Lepidoptera: Geometridae) in Zhejiang, eastern China have differentiated into different species. Acta Entomologica Sinica 57, 1117-1122.

Zhang, H.H. \& Tan, J.C. (2004) Tea pests and non-pollution management in China. Hefei, Anhui Science and Technology Publishing House, 1-389.

Zhang, G.H., Ysuan, Z.J., Zhang, C.X., Yin, K.S., Tang, M.J., Guo, H.W., Fu, J.Y., Xiao, Q. (2014) Detecting deep divergence in seventeen populations of tea geometrid (Ectropis obliqua Prout) in China by COI mtDNA and cross-breeding. PLoS ONE 9, e99373. 